\title{
Impact of modern instrumentation on the system of basic concepts in metrology
}

\author{
J.M. Jaworski, J. Bek, A.J. Fiok \\ Warsaw University of Technology, Warsaw, Poland
}

This is a reissue of a paper which appeared in ACTA IMEKO ACTA IMEKO 1988, Proceedings of the Proceedings of the $11^{\text {th }}$ Triennial World Congress of the International Measurement Confederation (IMEKO), "Instrumentation for the 21st century", 16-21.10.1988, Houston, pp. 125-134.

The paper is devoted to the new system of basic concepts in metrology which better fits to the development of contemporary instrumentation and measurement science. The existing concepts are confronted with the current needs; their modifications or new concepts are proposed. In particular the concepts of quantity and measurement method have been broadened and generalised. The proposed system is based on understanding of measurement as an experiment of parameter identification of the model of the measured object. One of the most important new concepts introduced here is the concept of measuring metasystem which comprises not only instrumentation but also the measured object, a "measurement interface" between the object and instrumentation and the operator.

\section{Section: RESEARCH PAPER}

Keywords: metrology, measurement model, error

Citation: J.M. Jaworski, J. Bek, A.J. Fiok, Impact of modern instrumentation on the system of basic concepts in metrology, Acta IMEKO, vol. 3, no. 1, article 10, May 2014, identifier: IMEKO-ACTA-03 (2014)-01-10

Editor: Luca Mari, Università Carlo Cattaneo

Received May $1^{\text {st }}, 2014$; In final form May $1^{\text {st }}, 2014$; Published May 2014

Copyright: (C) 2014 IMEKO. This is an open-access article distributed under the terms of the Creative Commons Attribution 3.0 License, which permits unrestricted use, distribution, and reproduction in any medium, provided the original author and source are credited

\section{INTRODUCTION}

Every field of human activity has its own system of basic concepts. If the development of a discipline has been relatively slow and uniform, then its system of basic concepts is complete, well-ordered and well-defined. Usually, fast progress in the discipline destroys completeness and orderliness of that system; some concepts lose their sense, the sense of many others has to be changed and there is a need for introduction of new ones.

In the last decade the progress in measurement science has been generally very significant and fast, but not uniform. The greatest and most rapid it has been in instrumentation mainly due to:

(i) proliferation of measurement needs in all the fields of science and technology,

(ii) increase of complexity of measurement tasks to be performed in practice,

(iii) great advances in technology, mostly in electronics (microprocessors, LSI and VLSI circuits and computers) which make possible implementation of instruments and systems realizing automatically nearly all physical and mathematical operations which are necessary in measurement practice.
New instruments (e.g. dynamic signal analysers, digital oscilloscopes) have introduced in metrology changes of not only quantitative character, but also of qualitative one (new types of measurements e.g. of spectrum and cepstrum) measurements giving deeper knowledge about the measured object than it was possible before.

In the paper the authors have tried to prove that the existing system of basic metrological concepts does not correspond to the contemporary state and expected development of instrumentation and up-to-date level of theoretical knowledge. The new system is necessary in everyday work of all metrologists; designers and users as well as teachers of measurement and instrumentation at all levels of education.

As the reference for our considerations we have the "International Vocabulary of Basic and General Terms in Metrology" [1] which is one of the latest texts dealing with the system of basic concepts. In the paper general rules of creation of a complete system of basic concepts as well as definitions of the most important new concepts and new definitions of some old concepts have been proposed. These proposals have been based both on the own experience of the authors and on the review of up-to-date technical literature, conference proceedings and catalogues of modern instrumentation. 


\section{AN OUTLINE OF A DEVELOPMENT OF INSTRUMENTATION AND MEASUREMENT METHODS}

In the development of measuring instruments and measurement methods three partially overlapping periods may be distinguished.

First there was a period of mechanical instruments and direct-comparison methods of measurement. The overwhelming majority of instruments were deflection type e.g. pressure gauge, rotameter, liquid thermometer, electromechanical voltmeter. Typical measurements by direct comparison methods were measuring of length by means of a gauge, of angle by means of a protractor, of voltage by means of a potentiometer or measurement of resistance by means of an electrical bridge.

In the second period electrical and electronic instruments and conversion-based measurement methods were the dominant ones. Measuring transducers enabled one to measure non-electrical quantities using electrical methods. Electronic instruments became the most important in measurement at first of electrical and next also other quantities. In the culmination point of that era digital instruments were introduced into everyday use.

The third stage of instrumentation development, closely connected with the "electronic" one, is the stage of computerization. The dominant measurement methods are indirect ones based on computerized signal processing and calculations. The computer becomes the "heart" of a measuring instrument or a system; it controls the whole measurement process and processes the data carried by the signals from the sensors. The function of traditional readout devices has been taken over by monitors. Knowledge about designing measurement algorithms and computer programming becomes of major importance for anybody who wants to measure.

\section{CONTEMPORARY TASKS OF METROLOGY}

In classical metrology a measurement has been mainly treated as an experimental determination of the value of a quantity. But in everyday practice we are mainly interested in obtaining a quantitative information about the state or properties of the given object, while a physical quantity accessible for instruments is only a carrier of the required information. The modern instrumentation offers new technical facilities enabling us to extract this information. To achieve it, the tasks of modern metrology have been extended in comparison to the classical one. Contemporary measurements are aimed at determining not only the value of a quantity but $[2$, 3] also:

- variation of a physical quantity with time, e.g. its temporal distribution or "waveform",

- distribution of a quantity in space, e.g. in measurement of surface geometry,

- mathematical representations of quantities, e.g. spectral density,

- relations between quantities, e.g. current-voltage characteristic of an electric element, their distributions or representations,

- parameters of such relations, e.g. attenuation.

Moreover, in many cases we do now measurements which differ from the traditional ones in their basic general character. To such "new types" of measurements belong:

(i) complex concurrent measurements of several interrelated quantities which through the relations between these quantities characterize the state and properties of the investigated object; this aspect of contemporary measurements is sometimes called "complexity of measurement",

(ii) measurements in which obtaining of information about the investigated object requires exciting it with an appropriate stimulus and sensing a response signal [2, 3, 6]; such measurements, common in investigation of many electrical, mechanical, optical and physico-chemical properties of great variety of measured objects can be called "active" measurements,

(iii) measurements which include operations typical for diagnostics, quality control, image recognition, model identification, a.s.o.

\section{ASSUMPTIONS OF THE PROPOSED SYSTEM OF BASIC CONCEPTS OF METROLOGY}

In the result of analysis of the contemporary measurements we decided to propose a new system of basic concepts in metrology. Let us mention some important assumptions of the proposed system. Structure of the system of basic concepts in a particular science should correspond with the structure of this science. We have assumed that $[2,5]$ metrology or using other words - science of measurement and instrumentation - can be divided into four parts: theory, technique, instrumentation and legislation.

Theory of metrology (we shall call it measurement theory) is the system of general laws of metrology.

Technique in metrology (we shall call it measuring technique) includes the knowledge concerning purposeful, usually theorybased, activity connected with planning, organization and execution of measurement as well as evaluation, verification and interpretation of measurement results.

Instrumentation connotes all the knowledge on measuring instruments, systems end devices.

Legislation (i.e. legal metrology) is a system of law regulations concerning measurements.

We proposed to group the basic concepts into six parts:

1) Fundamentals of metrology

2) Measurement and its component operations

3) Errors

4) Measuring instruments and their properties

5) Measuring technique

6) Legal metrology

Up to now our works have been concentrated on the first three parts and the fifth one. So the concepts dealing with measuring instruments and legal metrology have not been considered in this paper.

The proposed system covers in principle the concepts connected with measurements in exact sciences (first of all in physics and technology). For other fields like biology, psychology or economics, perhaps some modifications of the system may be needed.

Our considerations have been aimed at the system of concepts. The concepts are of primary importance; the terms (names of concepts) are also of importance, but in the stage reported here, our work has been concentrated on the concepts, not on the names of them. Of course it has been necessary to use names, but in a lot of cases they are only tentative ones; in some cases no existing word fits with the needed meaning, so we have tried to propose neologisms.

The classical approach to the metrology has assumed that the subject of measurement is a physical quantity [1]. The basic assumption of our approach is that the subject of measurement 
is a physical object and measurement is treated as an experiment of parameter identification of the model of the measured object $[2,3,5,6]$.

\section{MEASURED OBJECT, ITS MODEL, QUANTITIES}

Classical metrology defines [1] measurement as "the set of operations having the object of determining the value of a quantity" and quantity as "an attribute of phenomenon, body or substance, which may be distinguished qualitatively and determined quantitatively". Many metrologists consider quantities as real entities being the objects of measurement or experiment. In fact, quantity understood in classical sense is an abstract concept defined for idealized (abstract) objects, different from real objects which are to be measured.

In our opinion no measurement can be separated from a part of objective reality - a physical object (called measured object) whose chosen properties are to be quantitatively determined in effect of the measurement.

A quantitative determination is a determination by means of mathematical categories. The categories describing properties of the object must be exactly defined. To do it we must choose, assume or create a mathematical model of the measured object. Measurement-oriented mathematical model of the measured object is a mathematical or equivalent (e.g. circuit-type) description of all its properties and relations between them which are relevant to the measurement task.

The concept of quantity is and will remain one of primary concepts in metrology and in mathematical modelling of physical objects. We propose only to broaden and generalize this basic concept.

Our approach takes into account that:

(i) generally, quantity itself is not a property of real objects; it only models its particular property within bounds of the assumed mathematical model of the object,

(ii) temporal and/or space aspects of object properties can be of major importance,

(iii) it is necessary to distinguish clearly different concepts named with the same term "quantity".

In classical sense quantities are defined as families of some properties of idealized entities abstracted from relations of equivalence - verifiable by idealized experiments. For instance, Ajdukiewicz [8] gives the definition of length: "length is a family of ideal properties abstracted from the relation of congruence of line sections". The definition of quantity cited at the beginning of this clause given in [1], common in classical metrology, has the same character and fails in many practical measurement situations. For example it is not too easy to utilize the above definition of length, very good for "measurement" of abstract object (line section), in measurement of real 3dimensional object as a cylinder.

Real objects are always "imperfect" in comparison with abstract objects for which physical quantities (in classical meaning) have been defined. In our opinion quantities should be treated as elements of assumed mathematical model of measured objects. In this sense quantities model elementary properties of the object and of phenomena in it. To distinguish clearly two meanings of the term, we will call quantities being part of the model of real object "modelling quantities" in contrary to those defined for abstract objects called by us (it necessary) "abstract quantities".

Mathematical model of the object consists in general of:

(i) set of modelling quantities, (ii) set of equations which model relations between properties of objects and phenomena in it (we shall call them model equations).

Sometimes model may consist of modelling quantities only.

The set of modelling quantities and their mathematical character and type of model equations give the structure of the model. The coefficients of model equations are parameters of the model. It is possible to generalize the concept of model parameter as in [6].

The mathematical model of a physical object is always an approximation; if accuracy of the model is high (for the particular object measured for a given aim in given conditions) we may ignore this fact. But in some cases errors of this approximation can be of major importance from the point of view of accuracy of the whole measurement; sometimes the measurement results may be even completely without sense due to assumption of oversimplified model e.g. one dimensional (when measuring the diameter of a cylinder) or linear (when measuring the capacity of ferroelectric condenser in the range of high voltages).

For a particular class of objects it is possible to construct or choose a series of models of different accuracy. The utilized model should be optimized from the point of view of the particular measurement task: as simple as possible to make measurement easier and cheaper; as complicated as necessary to include all the factors which may preclude obtaining reliable measurement results of sufficient accuracy.

Different meanings of the term "quantity" which should be distinguished are as follows:

(i) quantity as a property of abstract objects (abstract quantity),

(ii) manifestation of a quantity (in an abstract sense) (perhaps it would be better to call it manifestation of a property [9]) i.e. a specific state of property; if two objects are equivalent in the sense of the property considered, they give the same manifestation of this property; the property can be treated as a set of manifestations and relations between them,

(iii) mathematical model of a quantity,

(iv) values of quantity manifestations, i.e. mathematical categories (e.g. numbers) which are mapping manifestations of the quantity; the mathematical model of a quantity is a set of these categories and relations between them (mapping relations between manifestations).

Unfortunately these different concepts are usually not distinguished by different terms; the term "quantity" is commonly used for the first three concepts and term "value of a quantity" for the concepts (ii) and (iv).

The world of physical quantities (treated as properties of physical objects) given by the classical metrological system of quantities is very simplified; it neglects temporal and spatial aspects of properties of objects. The quantities defined in this world do not depend on the time and space coordinates; we shall call them the static-lumped quantities.

Such concept of static lumped quantity should be generalized. We propose to introduce concepts of a staticdistributed quantity, a dynamic-lumped quantity and a dynamicdistributed quantity. Such generalized quantities are also attributes of objects. Their manifestations are mapped into different mathematical categories. Images of manifestations (we shall call them values of a quantity, spatial distributions of a quantity, temporal distributions and spatio-temporal distributions) are real numbers, functions of the spatial coordinates, functions of time and functions of time and spatial coordinates. 
The next step in generalization of quantity is concept of the mathematical representation of quantity which is a transformation of quantity values or distributions into the domain of certain mathematical categories. Signal energy, Fourier transform, autocorrelation function are typical examples of representations.

Our system of basic concepts defines also concepts of: measurement scale, additive quantity, interval quantity, vector quantity, unit of quantity, system of quantities, system of units, basic quantity etc.

\section{MEASUREMENT AND ITS COMPONENT OPERATIONS}

Classical definition of measurement [1] is: "the set of operations having the object of determining the value of a quantity". The quantity in the definition is "static lumped", its values are real numbers.

We would like to change the definition to: "measurement is the experiment carried out upon a physical object and aimed at determination of:

(i) values or distributions of quantities modelling an object,

(ii) values of mathematical representations of quantities modelling an object,

(iii) relations between quantities modelling an object or between representations of these quantities".

The term "quantity" used in the proposed definition means a quantity in generalized sense.

Quantities, their representations, relations between quantities or representations (the relations may be given by parameters of the standard equations), mentioned in definition, are measurands. The fact of great importance for practice is that "values" of these measurands may be not only real numbers but also other mathematical categories as complex numbers, functions of any kinds, sets of numbers, etc.

The definition proposed above is closer to "identification" concept of measurement then the classical one. If we only broaden the meaning of parameters of model, treating the values and distributions of quantities or their representations (if they are measurands) and the specific forms of relations between them as values of parameters of the model, then we can define measurement as "an experiment of parameter identification of mathematical model of an object to be measured" [6, 7].

This approach to measurement has many theoretical and practical consequences. The most important ones of them seem to be [2]:

(i) Widespread interpretation of measurement as a reliable method of objective cognition of reality is no more strictly valid. By means of measurement we investigate reality but only within the bounds of accepted structures of its mathematical models.

(ii) The parameter identification approach to the measurement enables one to reinterpret the problem of "true value" as an absolute aim and reference of measurement.

(iii) This approach to measurement entails relevant changes in measuring technique, what will be discussed later on.

In the classical model of measurement shown in Figure 1 measurement is considered as consisting mainly of operation of comparison of the measured quantity (or a quantity dependent on the measured quantity) with the standard quantity of known value. Much less attention has been paid to other operations: sensing of the signal from the measured object (in most cases the concept of measurement signal was not utilized or the signal was treated as the same as the measured quantity itself), conversion (in most cases aimed on signal conditioning) and

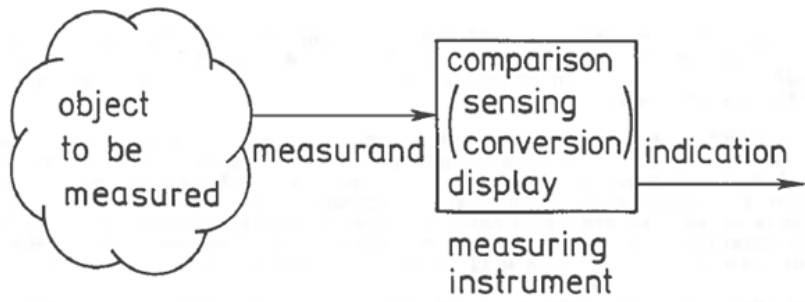

Figure 1. Classical model of the measurement.

display of measurement result.

In engineering practice measurements of complex objects aimed at determination of many measurands are very often executed at present and in most of cases the measurands are not directly accessible for measuring instruments. One of many examples are measurements of magnetic materials.

Contemporary measuring instruments and systems require use of a modified model of measurement. The model shown in Figure 2 seems to describe well modern measurements [7]. The "inputs" of a measuring instrument (or a system) are measurement signals generated by the measured object or exciting the object. Exciting (test) signals, even if generated within the instrument, are from the informative point of view the inputs for the instrument. The measurement signals are in general multidimensional ones.

The measured object is described by its mathematical model. The structure of the model (the set of modelling quantities and the type of model equations) is given a priori, the model parameters (in the generalized sense) are the measurands.

The measuring system accomplishes first of all operations of sensing and comparison. The operation of sensing is often associated with operation of actuating the measured object with the appropriate stimulus (test signal). The other basic operations in the system are: signal and information processing (enhanced version of classical conversion covering all computerized data processing) and formation of an output signal whose particular case is visualization of the results.

The purpose of signal and information processing is the determination of measurands based on the information carried by the measurement signals. To accomplish this task the relations between the measurands and the measurement signals must be strictly determined what is equivalent to the acceptance of the model of the measurement interface between the object and the system. Both the structure of the object model and the structure

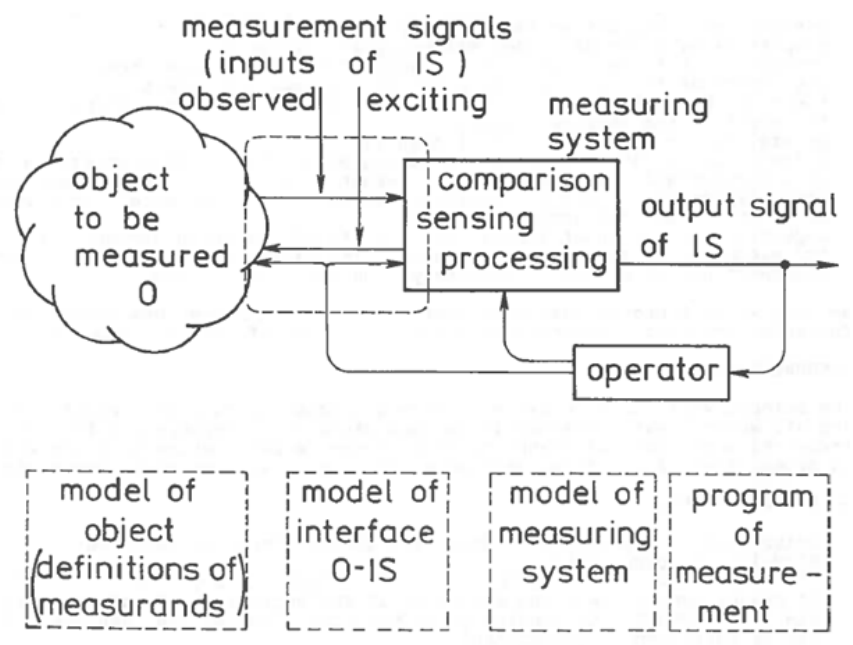

Figure 2. Model proposed for modern measurements and illustration of the concept "measuring metasystem". 
and parameters of an interface model are decided on the base of a-priori knowledge about the object and the system. The same is true in relation to the model of measuring system.

In fact each description of metrological properties of measuring instrument or system of instruments is its model. Mathematical model of classical instrument was sometimes so simple that in some cases it was possible to give it on the front panel of the instrument. Model of contemporary instrument is more complex, it needs many pages of the text to be given. The structure and parameters of the model of measuring instruments or system must be known to its user before the execution of the measurement.

The classical metrology has put the main stress on the operation of comparison; measurement methods have been distinguished mainly from the point of view of the way of comparison used (e.g. direct-comparison method, substitution method and null-method [1]).

Our system of basic concepts saves these concepts but takes into account that the measurement comprises also other operations and the measurement method is determined not only by the method of comparison but also by the methods of signal sensing, signal conversion as well as the method(s) of signal and information processing.

Each operation utilized in the measurement can have "own" methods of realization of its task and some of them should be included in the system of concepts. Functioning of contemporary instruments, especially computerized and intelligent ones, is based on sensing and processing of signals. Classical metrology paid a very little attention to informative and signal aspects of measurement. So it is necessary to define - from the point of view of metrology - several general concepts (e.g. measurement information, measurement signal, parameters of a measurement signal) as well as some specific concepts connected with forms of measurement signal, types of carried information, types of signal conversion etc.

The classical model of measurement shown in Figure 1 is a particular, very simplified case of the proposed more general model which was discussed above. In the classical model of measurement the model of measured object is reduced to the quantity modelling the object.

\section{ERRORS}

The insufficiency of the set of concepts connected with errors in measurement considered by classical metrology seems to be now evident.

The classical error theory deals only with the inaccuracy of measurements whose results are real numbers. Results of contemporary measurements may be also complex numbers, series of real numbers or complex ones, functions (both real and complex ones) etc., as well as parameters of functions or equations. The new set of concepts should also refer to such situations.

Each operation accomplished in a measuring system is associated with errors, because some of these operations were not considered by classical metrology, these errors have not been considered.

For our system of basic concepts new concepts in the error theory have been needed. An attempt to formulate a broadened and modified system of concepts of error theory has been presented in [4]. The most important elements of the system are:

1) The word "error" has two meanings:

- an event of discrepancy between two entities compared, the first one being the reference for the other, the other being the realization of the first one,

- a mathematical model of the discrepancy which represents it in mathematical categories.

2) In metrology, compared entities (measurands, signal parameters) are mathematical categories or have their own mathematical models so that the error is determined by comparison of mathematical categories in a proper space of compared categories (of e.g. complex numbers, functions of given type etc.). There are two main kinds of errors:

- difference error defined by means of algebraic difference between categories compared e.g. "classical" absolute and relative errors of measurement [1],

- metric error defined by means of "the distance" (in mathematical sense) between categories compared e.g. mean square error as a measure of a discrepancy between two functions.

Errors in the domain of real numbers remain very important; their field of application is broad and in many cases it is possible to reduce other types of errors to this type.

3) Two kinds of errors as mathematical models must be strictly distinguished:

- true error (more precisely conventionally true error) defined as mathematical model of discrepancy between two categories compared,

- boundary error defined as the parameter (or parameters) determining a subspace (in space of categories compared) which surrounds one of the category compared and contains the other one (or for which there is a probability large enough that it may contain the other category).

In classical metrology reference is a true value; in metrology based on identification concept of measurement in many cases reference can be given by a model more exact than the used one.

4) Set of errors distinguished in classical metrology should be broadened. Among others there is a need to take into account also:

- error of the model of measured object,

- error of the model of interface between the object and the measuring system,

- error of data processing.

\section{MEASURING TECHNIQUE}

Measuring technique is understood as the system of reasonable and theoretically based methods of carrying out measurement.

Our system of basic concepts connected with measuring technique bases on two concepts: measuring process and measuring metasystem [4].

Measuring process is a connected series of actions and operations deliberately undertaken to carry out given measurement.

Up to now the concept of measuring system has been limited to "a complete set of measuring instruments and other equipment assembled to carry out a specified measurement task" [1]. We would like to broaden the concept to embrace (Figure 2) all the things and ideas needed to carry out given measurement i.e.:

(i) object to be measured,

(ii) mathematical model of the object, exactly the structure of model and definitions of its parameters which are measurands for measurement,

(iii) set of measuring instruments, its mathematical model and programs (instrumental part of the system), 
(iv) mathematical model of the interface object - instrumental part of the system,

(v) program of measurement,

(vi) operator.

To avoid confusion of the new meaning of the term "measuring system" with the traditional one, we propose to introduce the new term "measuring metasystem" for the measuring system understood in the new broadened sense. The instrumental part of the metasystem might be called, as before "measuring system".

Measuring process has some typical stages. We can group them in three main parts of this process:

- preparation of measurement,

- execution of the measurement,

- postprocessing of measurement results.

The object to be measured is an operand for measurement. Specification of the measurement is a starting point of measuring process. The specification above all consists of:

- mathematical model of the object to be measured, exactly the structure of model and definitions of measurands,

- permissible errors of measurement,

- permissible costs and time of the measurement.

In typical situation specification is given a priori but in practice it may be necessary to correct elements of specification.

The main parts of measuring process may be subdivided, e.g. the first part, preparation of measurement, into:

- creation of the conception of measurement (fixing model of interface object-instruments, choosing the methods of signal sensing, conversion and processing)

- design of measuring system and of measurement program,

- implementation of measuring system,

- making the measuring system ready for proper functioning (including the identification of the mathematical model and errors of the system).

The characteristic feature of the modern measuring process seems to be a need of validation and correction of partial results. The effect of each operation in measuring process has to be examined from the point of view of its validity and congruency with the preliminary specification. The needed correction of effect of each operation may be accomplished by a proper modification of this operation and, if necessary, of the previous ones. In a limit case even a change of elements of the measurements specification (e.g. of the object model or of requirements dealing with errors or costs) may be necessary.

The broadening of the concept of measuring system is necessary not from the theoretical but from the very practical point of view. It is an effect of the real situation existing in contemporary measurements. It is now impossible to separate hardware from software in modern instrumentation and an interaction (both in hardware and software sense) between the measured object and the measuring system is of major importance.

\section{SUMMARY AND CONCLUSIONS}

Let us underline once more that the aim of our work reported here is rather practical; we would like to work out the theoretical tools which could be useful for designers and users of modern instrumentation and would facilitate their everyday work.

We did not intend to present in this paper a complete system of basic concepts in metrology. It is impossible because creation of a new system is not a trivial task; it requires much effort, profound discussions with the outstanding experts in measurement science and instrumentation. The work has not been completed yet. We presented here only some proposals concerning the scope and the selected most important concepts connected with chosen parts of the system. Your valuable opinion will be of major importance for our further work. The most important elements of our approach to the system of basic concepts in metrology are:

- observation that the aim of measurement is to obtain not the value of an abstract quantity but an image of the measured real object mapping its properties by means of proper mathematical categories,

- conclusion that one of the first stages of the measurement process must be the choice of the mathematical model of the measured object,

- treating the measurement as an experiment of parameter identification of the model of the measured object,

- generalization of the concept of quantity,

- introduction of the concept of measuring metasystem which consists not only of instrumentation but also of: the measured object, the measurement interface between the object and instruments, the whole "software" (in a very broad sense) and the operator,

- understanding the major importance of parts of measuring process prior to the execution of the measurement and of the validation of effects of each component operation and, if necessary, corrections of them.

We hope we have proved that contemporary measurements need new system of basic concepts. The system reported in the paper is one of possible proposals.

\section{ACKNOWLEDGEMENTS}

The authors would like to express their gratitude to many colleagues - metrologists whose views presented in various discussions seriously influenced the presented work. Special thanks to Prof. Roman Morawaki whose opinions and help in preparation of the final version of this paper were of great importance.

\section{REFERENCES}

[1] International Vocabulary of Basic and General Terms in Metrology, BIPM, IEC, ISO, OIML, 1983.

[2] A.J. Fiok, J.M. Jaworski, R.Z. Morawski, J.S. Oledzki, A.C. Urban,Theory of measurement in teaching metrology at the engineering faculties, Proc. 8th Int. IMEKO-TC1 Colloquium on Higher Education, Warsaw, 1986.

[3] A.J. Fiok, J.M. Jaworski, What to teach in measurement and instrumentation, 8th Int. IMEKO_-TC1 Colloquium on Higher Education, Warsaw, 1986.

[4] J.M. Jaworski, J. Bek, Theory of errors in metrology. an attempt to formulate the system of basic concepts, 10th IMEKO Congress, Prague, 1985, Vol. 1, pp. 124-130.

[5] J. Bek, A.J. Fiok, J.M. Jaworski, J.S. Oledzki, Structure of metrology and its system of basic concepts, Pomiary, Automatyka, Kontrola, 1987, No. 8, pp. 175-177 (in Polish).

[6] J.M. Jaworski, A new parameter model of measurement. Proc. 5th Int. IMEKO-TC7 Symposium on Intelligent Measurement (ed. by D.Hofmann), Jena, 1986, Vol. 2, pp. 250-252.

[7] J.M. Jaworski, Errors, noise and disturbances in measuring system, 1st Int. IMEKO-TC4 Symposium on Measurement, Como (Italy) 1986, pp. 27-32.

[8] K. Ajdukiewicz, Pragmatic logic, PWN, Warszawa 1985 (in Polish).

[9] L. Finkelstein, Theory and philosophy of measurement, Chapter 1 in: Handbook of Measurement Science ed. by P.Sydenham, J.Wiley, Chichester, New York 1982. 